\title{
I LAICI NELLA CHIESA COME COSTRUTTORI DI COMUNIONE: LA CHIESA ANTICA
}

\section{LAY PEOPLE IN THE CHURCH AS BUILDERS OF COMMUNION: THE ANCIENT CHURCH}

The study begins with a brief history of the formation of the concepts and terms of "clergy" and "laity" in the Church of the first centuries, then moving on to analyze the case, considered exemplary, of the relations between the Egyptian monasticism of the IV-V century (lay) and the institutional Church (episcopate). In a situation of strong ecclesial divisions, due to the spread of Christological heresies and the consequent political and ecclesial struggles, the cultural operation implemented by Athanasius of Alexandria meant that the strong popularity and the deep charismatic nature of the lay monastic movement was turned in a valid support to the institutional Church, developing forms of collaboration and complementarity and leaving aside opposing identities and practices. Similarly, albeit with due distinctions, the study analyzes briefly the realities of contemporary ecclesial movements and the resources they represent for the pastoral and missionary potential of the Church in our day. Also in this case, a correct relationship between charism and institution is necessary, not conflicting but complementary and mutually respectful..

Key words: clergy, laity, Church of the first centuries, patrology. 
Per una migliore comprensione della vocazione dei laici all'interno del popolo di Dio farò innanzitutto una breve introduzione storica circa il termine "laico" per analizzare poi il ruolo dei laici nella Chiesa antica. ${ }^{1}$

\section{La cultura giudaica e neotestamentaria}

Nel Nuovo Testamento, come nella Bibbia ebraica i laici, ovviamente, sono presenti, anche se nella Scrittura il termine «laico» nel senso con il quale noi lo intendiamo non esiste. La società ebraica veterotestamentaria era caratterizzata da una netta separazione fra il ceto sacerdotale e regale e il resto del popolo. I primi ricevevano l'unzione, di carattere dinastico che li separava nettamente dal resto del popolo. Del popolo facevano parte profeti, figure forti che oggi diremmo laicali, così come furono laici anche tutti i patriarchi, da Noè ad Abramo, a Isacco, a Giacobbe e molti altri grandi personaggi della storia di Israele. Anche Mosè, al quale Dio affidò l'edificazione concreta del proprio popolo per mezzo dell'Esodo, può essere considerato un laico, come i profeti.

Lo stesso Cristo apparteneva alla tribù "laicale" di Giuda. A tal proposito la lettera agli Ebrei afferma: "Se Gesù fosse sulla terra, egli non sarebbe neppure sacerdote" (Eb 8,4), evidentemente in senso legale e secondo gli schemi ebraici.

Nel Nuovo Testamento, assieme a Gesù erano «laici» Maria sua madre e lo sposo Giuseppe. Il Signore si manifestò per primi ai pastori (laici), poi ai magi dell'Oriente (laici) e compì il suo primo miracolo a Cana, in una festa matrimoniale e, dunque, in un contesto tipicamente laicale. Si può aggiungere che verso il Cristo il sacerdozio del suo tempo non si dimostrò certamente accogliente. E laici, ovviamente, erano anche i dodici apostoli, fino alla loro partecipazione all'ultima cena, quando fu loro chiesto: «Fate questo in memoria di me».

Le lettere di Paolo hanno tutte per destinatari i cristiani "gentili", da considerare nel loro complesso laici e lo stesso si può dire delle altre Lettere cattoliche. ${ }^{2}$

Per una bibliografia su questo vasto tema si può vedere: Y.-M. Congar, Laïc et laïcat, in Dictionnaire de Spiritualité, IX, Paris 1976, coll. 79-108; A. Scola, C. Giuliodori, et al. (a cura di), Il laicato. Rassegna bibliografica in lingua italiana, tedesca, francese, LEV, Città del Vaticano 1987; N. Ciola, Indicazioni bibliografiche sulla teologia del laicato, in Orientamenti Pastorali, 34 (1986), pp. 79-82; R. Goldie, Laici, laicato, laicità. Bilancio di trent'anni di bibliografia, Ed. AVE, Roma 1986.

2 Il Concilio Ecumenico vaticano II ha sottolineato questo carattere "laicale" della Chiesa apostolica nel suo Decreto sull'apostolato dei Laici Apostolicam Actuositatem del 18 novembre 1965, che al n. 1 afferma: "L'apostolato dei laici, infatti, derivando dalla loro stessa vocazione cristiana, non può mai venir meno nella Chiesa. La stessa sacra Scrittura mostra abbondantemente quanto spontanea e fruttuosa fosse tale attività ai primordi della Chiesa" (cfr. At 11,19-21; 18,26; Rm 16,1-16; Fil 4,3)." 
Per quanto riguarda il termine "laico" esso deriva dalla traduzione greca dei Settanta dall'ebraico (avvenuta nei secoli III-II a.C.) dell'espressione popolo, così pregnante per l'Antico Testamento, con il termine laòs, nel senso, però, di "popolo di Dio", nome collettivo dell'intero Israele. Da qui è venuto l'aggettivo latino laicus usato una sola volta nella Vulgata di S. Girolamo (traduzione dal greco del IV secolo). ${ }^{3}$

\section{Nella Chiesa delle origini}

Nella sua prima Lettera, scritta in greco, san Pietro aveva raccomandato ai "presbiteri" (in greco presbyteroi, i più anziani) delle comunità dell'Asia Minore, "pascite qui est in vobis gregem Dei providentes non coacto sed spontanee secundum Deum neque turpis lucri gratia sed voluntarie neque ut dominantes in cleris sed formæ facti gregi et ex animo", ${ }^{4}$ vale a dire "pascete il gregge di Dio che vi è affidato, sorvegliandolo non per forza ma volentieri secondo Dio; non per vile interesse, ma di buon animo; non spadroneggiando sull'eredità a voi affidata, ma facendovi modelli del gregge". Nella Vulgata S. Girolamo aveva reso col termine latino clerus la parola greca klèros, che significa appunto eredità. Avvenne così che la raccomandazione di Pietro portò alla realizzazione del suo esatto contrario: il «clero», appropriandosi di questo termine, in qualche modo si appropriò anche del suo contenuto, pretendendo una specie di esclusività dell' "eredità-klèros" di Gesù.

Circa mezzo secolo più tardi, intorno all'anno 96, papa Clemente, quarto vescovo di Roma, scrisse anch'egli una lettera alla comunità dei cristiani di Corinto, dove erano avvenuti forti contrasti fra clero e laici (termini, questi, che ancora non esistevano). ${ }^{5}$ In questo testo, che la critica ha stabilito essere pseudoepigrafo, egli adoperò per la prima volta al Cap. 5 la parola greca laikòs, da laòs, per indicare i cristiani comuni, i "non presbiteri" per distinguerli dal klèros. ${ }^{6}$ A questo nuovo termine egli diede un contenuto che, almeno di fatto,

\footnotetext{
3 Cfr. P. Neuner, Per una teologia del popolo di Dio, Queriniana, Brescia 2016, p. 34-ss.

4 Vulgata, Epistula I Petri, 5, 1-2.

5 Cfr. Didachè. Prima lettera di Clemente ai Corinzi. A Diogneto, Roma, Città Nuova 2008, pp. 7-12. Testo italiano pp. 29-77.

6 Per un'analisi approfondita del diverso ruolo e ministero dei laici e del clero in questa lettera si veda A. Faivre, Les Fonctions ecclésiales dans les écrits Pseudo-Clémentins, in "Revue de Sciences Religieuses", 50, Strasbourg 1976, pp. 97-111.
} 
sanciva questa netta divisione del popolo di Dio in due categorie non più di uguale dignità. ${ }^{7}$

In un altro passo, il Cap, 14, lo Pseudo-Clemente racconta di essere stato ordinato vescovo da Pietro e riferisce gli insegnamenti da lui ricevuti. Tra questi la raccomandazione di lasciare ai laici l'impegno nelle cose del mondo e che i presbiteri non si occupino in queste. Seguono la descrizione della Chiesa come «una grande nave che attraversa un mare procelloso» e la seguente affermazione:

«Ma è soprattutto importante che i passeggeri, ossia i laici, se ne stiano quieti e silenziosi, ciascuno al proprio posto, così da evitare che, con la loro agitazione e con i loro movimenti confusi e inutili, spostandosi qua e là finiscano per ostacolare le manovre dei marinai o che, rilassandosi per irrequietezza su un solo lato della nave, questa, sbilanciata, vada a fondo.» ${ }^{8}$

Inizia così una sorta di processo di separazione, se non addirittura contrapposizione, fra i due gruppi dei laici e del clero che proseguirà fino ai giorni nostri con fasi alterne. Non mi addentro sulle evoluzioni successive che, specialmente a partire dal Concilio Vaticano II, hanno radicalmente modificato il concetto, il ruolo e, specialmente, la vocazione dei laici all'interno del popolo di Dio.

Ma restiamo alla Chiesa antica, a cui sono stato chiamato a limitarmi.

\section{Un esempio di laicato nella chiesa antica: il monachesimo}

Quando si pensa al laicato nella chiesa antica non si può pensare solo ad una massa popolare informe e senza fisionomia. Esiste un settore particolarmente significativo che svolse un ruolo ben definito, e cioè il monachesimo.

Esso fin dalle origini mantiene ed esalta il proprio carattere laicale. I monaci rifiutavano infatti il proprio coinvolgimento tanto nel presbiterato che nell'episcopato. ${ }^{9}$ Mentre nel monachesimo prevaleva la funzione di "padre" e, di conseguenza, giocavano un ruolo determinante le doti spirituali acquisite mediante un lungo esercizio ascetico, nel sacerdozio ed episcopato aveva la preminenza la funzione di "maestro" la cui origine risiede soprattutto nella grazia sacramentale collegata alla preghiera consacratoria. Questo spiega la diversa prospettiva con la quale i due ruoli venivano percepiti e, di conseguenza,

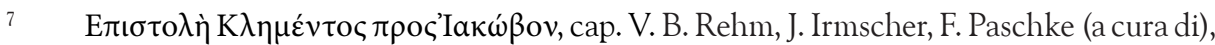
Die Pseudoklementinen I. Homilien, $2^{\text {nd }}$ ed., (Die griechischen christlichen Schriftsteller 42), Berlin, Akademie-Verlag 1969, p. 5-22.

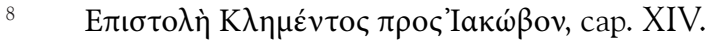

9 Cfr. P. Neuner, Per una teologia..., cit. pp. 45-47. Su tale argomento Giovanni Cassiano affermava: "Un monaco dovrebbe in ogni modo evitare le donne e i vescovi." Istituzioni Cenobitiche XI, 17. 
valutati in base ad una scala di valori all'interno dalla quale l'umiltà era elemento fondamentale per i monaci. ${ }^{10}$

Un'autorevole conferma di tale realtà ci giunge da Atanasio che nel 354 scrive a Draconzio, monaco da lui ordinato vescovo e che, dopo un periodo di esilio impostogli dal patriarca ariano Giorgio, preferisce tornare alla sua primitiva forma di vita monastica piuttosto che riprendere la guida della sua diocesi. ${ }^{11}$ Si ricava chiaramente dal testo che Draconzio è trattenuto da due ordini di obiezioni: ${ }^{12}$ il primo è l'esercizio dell'umiltà, connaturato alla spiritualità monastica, intesa come senso di indegnità a ricoprire cariche che in qualche modo comportassero un ruolo di guida di altri uomini; il secondo è il rifiuto a rinunciare alla pratica di una rigida ascesi, ritenuta inconciliabile con l'esercizio del ministero presbiterale o, ancor di più, di quello episcopale. ${ }^{13}$

Questo però non significava che i monaci si disinteressassero della sorte del popolo cristiano e rinunciassero a svolgere compiti che noi oggi diremmo di "pastorale". Il ruolo infatti svolto dai monaci egiziani nell'evangelizzazione degli ambienti pagani e il loro frequente intervento in favore dei cristiani dei

$10 \quad$ "Both monks and clergy claimed to teach with spiritual authority, but they were at variance with regard to the origin of their authority and the content, method, and medium of their teaching. The monks had prepared themselves through a lifetime of ascetism and deprivation to become vessels of the Holy Spirit. ... In contrast with the desert fathers, the priests and bishops derived their authority and their mission to teach from their ordination. ... Although it was often demanded that only truly spiritual men should become clerics, in practice the candidates for the clergy where, more often than not, recruited from among the well-respected and prominent members of the local community, ... they where well educated, of prosperous background, and enjoyed the respect of their fellow citizens." C. Rapp, Holy Bishops in Late Antiquity. The Nature of Christian Leadership in an Age of Transition, Berkeley 2005, pp. 103-104. Il tema è trattato ampiamente nelle pp. $100-152$

11 H. C. Brennecke, et al. (edd.), Athanasius Werke, vol. II, 8, Walter De Gruyter, BerlinNew York 2000.

12 Così si evidenzia anche in G. J. M. Bartelink, "Les rapports entre le monachisme égyptien et l'episcopat d'Alexandrie", in Alexandrina. Hellénisme, Judaïsme et christianisme à Alexandrie. Mélanges offerts au P. Claude Mondésert, Étitions du Cerf, Paris 1987, p. 369 e in G. M. Colombàs, Il monachesimo delle origini, Jaca Book, Milano 1984-1990, v. I, p. 90.

13 Ripetutamente la lettera esorta Draconzio a non prestare ascolto ai monaci che lo consigliano di non fare ritorno alla propria sede episcopale, cfr. Lettera a Draconzio (EpDr) 3: "Ti prego di non considerare tali cose, e non dar retta a coloro che suggeriscono tali cose", EpDr 5: "Sento che quelli ti suggeriscono queste cose all'orecchio, e ritengono, così facendo, di essere pii; invece se lo fossero veramente rispetterebbero piuttosto il ministero che prima Dio ti ha affidato", EpDr 8: "Non ti distolgano dunque i monaci, come se tu fossi stato costituito solo dai monaci, e non trovare scuse", EpDr 9: "Non obiettino tali cose coloro che consigliano te", EpDr 10: "E non dare ascolto coloro che suggeriscono cose diversamente da queste...", ecc... 
villaggi vicini ai centri monastici, testimoniato da numerose fonti, indicano infatti la loro disponibilità ad un impegno diretto di tipo pastorale e sociale. ${ }^{14}$

Quanto finora descritto nella Chiesa dell'Egitto del IV-V secolo sembra confermare una reciproca distanza dei due mondi: da un lato il laicato monastico, dall'altro i ministeri ordinati all'interno di un'organizzazione ecclesiastica fortemente istituzionalizzata e strutturata. I due ambienti sembrano percorrere itinerari diversi e nettamente distinti l'uno all'altro.

Ma attorno alla metà del IV secolo avviene qualcosa che rompe questo schema rigido. Nel 328 Atanasio divenne vescovo di Alessandria, il più importante centro cristiano assieme a Roma e Costantinopoli, di importanza primaria per lo sviluppo del dogma e le controversie cristologiche. Come è noto, Atanasio dovette affrontare innumerevoli conflitti dovuti alle alterne vicende che portarono gli scismatici meleziani ed ariani a prevalere sulle opinioni dell'Imperatore e del vescovo di Costantinopoli. A seguito di questi continui scontri Atanasio subì cinque condanne all'esilio, ma rimase sempre uno strenuo lottatore in difesa del dogma cristologico del Concilio di Nicea e operò un'attiva politica ecclesiastica per difendere il suo ruolo e, con esso, l'ortodossia dottrinaria.

In questa lotta egli utilizzò strumenti di "politica ecclesiastica", quali le nomine episcopali con le quali cercò di rafforzare il partito dei vescovi a sé fedeli e di accentuare lo stretto controllo che tradizionalmente il patriarca alessandrino esercitava nei confronti dei vescovi egiziani. ${ }^{15}$ Altra azione politica è quella di costruire un asse di collegamento diretto col vescovo di Roma, ecc...

Ma il capolavoro della sua azione di politica ecclesiastica, se possiamo dire, Atanasio lo realizzò operando un'impresa di profondo valore culturale e spirituale. Durante il suo terzo esilio, fra il 356 e il 362, egli entrò in più stretto contatto con gli ambienti monastici dell'Alto Egitto. Il Grande Antonio, figura di notevole spessore ascetico e spirituale era da poco scomparso, e grazie anche

14 Si vedano a tal proposito: E. Wipszycka, La christianisation de l'Égypte aux IVe-Ve siècles: aspects sociaux et ethniques, in ead., Études sur le christianisme dans l'Égypte de l'antiquité tardive, (Studia Ephemeridis Augustinianum 52), Institututm Augustinianum, Roma 1996, pp. 64-105 ; W. H. C. Frend, I monaci e la fine del paganesimo greco-romano in Siria $e$ in Egitto, in P. F. Beatrice (a cura di), L'intolleranza cristiana nei confronti dei pagani, EDB, Bologna 1990, pp. 37-55.

15 E. Wipszycka, La christianisation de l'Égypte aux IVe-Ve siècles..., op. cit., p. 156: "In conclusione, possiamo dire che la formazione e lo sviluppo delle strutture fondamentali della Chiesa egiziana sono stati condizionati dal grande potere del patriarca, per ciò che riguarda i vescovati, e del vescovo, per ciò che riguarda le parrocchie. Solo quando era impossibile fare altrimenti, il patriarca e i vescovi egiziani si decidevano a delegare in parte il loro potere, e anche in questi casi si guardavano bene dal formare delle strutture stabili che rischiassero di diventare indipendenti. Questa tendenza all'assolutismo ecclesiastico aveva radici profonde in Egitto." 
alla sua azione carismatica e di paternità spirituale il monachesimo egiziano era giunto ad un grado di profonda maturazione. Il suo influsso ormai si estendeva a vastissime fasce della popolazione cristiana che riconosceva all'autorità carismatica dei monaci un ruolo decisivo. Ad essi fanno ordinariamente riferimento i cristiani egiziani per la direzione spirituale, la gestione delle vicende familiari e sociali più urgenti e gravose. È quanto emerge evidente, ad esempio, dai detti dei padri del deserto che riportano spesso le richieste dei cristiani che preferiscono fare ricorso all'insegnamento e al giudizio dei monaci, piuttosto che a quello dei sacerdoti o dei vescovi.

Atanasio intuisce in questa fase che per ampliare la sua influenza sul mondo ecclesiale egiziano non basta rafforzare gli aspetti istituzionali della chiesa, come gli episcopati, ma è necessario operare una saldatura con il forte carisma monastico. Egli compone pertanto in quegli anni la Vita Antonii, biografia del Santo abate del deserto, attraverso la quale offre un ritratto del monachesimo come espressione genuina ed autentica dell'ortodossia cattolica egiziana. ${ }^{16}$

Non sappiamo il grado di attendibilità storica dell'opera, quanto ci sia di Antonio e quanto di Atanasio in questo racconto biografico, ${ }^{17}$ ma di sicuro lo scopo dell'opera era quello di legare il proprio nome e il proprio ruolo istituzionale di capo della Chiesa egiziana all'espressione più autorevole della spiritualità del suo tempo, quella, appunto, monastica. Questa importantissima operazione culturale gli fornì un supporto più ampio e favorì la coesione della variegata realtà ecclesiastica alessandrina evitandone la parcellizzazione.

Per la prima volta, ed in modo autorevole e direi definitivo, il monachesimo, espressione del mondo laico che aveva una larghissima influenza sul popolo, non era più estraneo o contrapposto alla struttura istituzionale, ma si trovava la via per una collaborazione non solo di tipo funzionale ma direi di ordine spirituale profondamente radicato nel vissuto popolare. ${ }^{18}$

Ho voluto fare questo esempio per esprimere come l'opera geniale di un grande uomo di cultura e di fede quale Atanasio, così come anche la disponibilità dei monaci a spendere il proprio talento spirituale accumulato nel deserto per il bene della chiesa tutta, portò ad un risultato fruttuoso e duraturo di una

16 Versione greca: Athanase d'Alexandrie, Vie d'Antoine, a cura di G. J. M. Bartelink, (SC 400), Édions du Cerf, Paris 1994. Versione latina: H. Hoppenbrouwers, La plus ancienne version latine de la vie de S. Antoine par S. Athanase, Nijmegen 1960.

17 Cfr. B. Brennan, Athanasius' Vita Antonii, a Sociological Interpretation, in Vigiliae Christianae 39, Leiden 1985, pp. 209-227; M. Alexandre, La costruction d'un modèle de sainteté dans la Vie d'Antoine par Athanase d'Alexandrie, in P. Walter, Saint Antoine entre mythe et légende, Ellug, Grenoble 1996, pp. 63-93.

18 A tal proposito si veda anche: M. A. Williams, The Life of Antony and the Domestication of Charismatic Wisdom in Thematic Studies, Charisma and Sacred Biography, in The Journal of the American Academy of Religion 48, Oxford 1982, pp. 23-40. 
maggiore coesione e comunione ecclesiale che aiutò il superamento di quel periodo di forte crisi.

La Vita di Antonio infatti divenne ben presto un'opera fondamentale per la conoscenza del monachesimo orientale e portò alla sua diffusione anche in regioni lontane dall'Egitto. Senza il contributo culturale di Atanasio probabilmente il monachesimo egiziano non avrebbe assunto quel ruolo di esemplarità che rivestì nei secoli successivi, contribuendo non poco anche allo sviluppo del monachesimo occidentale.

\section{Dalla Chiesa di Atanasio e Antonio ai giorni nostri}

A questo punto mi piace fare un collegamento forse un po' audace, ma direi molto calzante fra questo episodio della vita culturale e spirituale della chiesa antica con quanto il Card. Ratzinger ebbe a dire nel 1998 in occasione del primo Congresso internazionale dei movimenti laicali che S. Giovanni Paolo II volle a Roma in occasione della Pentecoste. ${ }^{19}$

Anche in questo caso il periodo storico vedeva una certa distanza fra il "nuovo" mondo laicale più impegnato (i Movimenti) e le più antiche strutture ecclesiastiche istituzionali (Diocesi, Congregazioni, Parrocchie, ecc...). Una certa diffidenza reciproca faceva sì che i due mondi procedessero, come nel caso che abbiamo visto in precedenza, su due binari paralleli e poco comunicanti fra loro. Entrambe le realtà avevano una forte identità che faticava a trovare punti di convergenza e di collaborazione.

Il Card. Ratzinger, allora Prefetto della Congregazione per la Dottrina della Fede, da teologo raffinato e grande conoscitore della Chiesa, propose una sua originale sintesi culturale e spirituale che indicava un itinerario nei quali i due elementi, quello carismatico dei movimenti laicali e quello istituzionale, trovassero una reciproca complementarietà.

Egli disse a questo riguardo:

Le due parti devono imparare l'una dall'altra a lasciarsi purificare, a trovare la via che conduce a quei comportamenti di cui parla nell'inno alla carità Paolo (1 Cor $13,4 \mathrm{ss})$. Ai movimenti, quindi, va rivolto un monito: anche se nel loro cammino hanno trovato e partecipano ad altri la totalità della fede, essi sono un dono fatto alla totalità della Chiesa, e alle esigenze di questa totalità devono sottomettersi, per restare fedeli a ciò che è loro essenziale. Ma occorre che si dica chiaramente

$\overline{19}$ Sulla portata e il significato dell'evento si veda: C. Hegge, Il Vaticano II e i movimenti ecclesiali: una recezione carismatica, Città Nuova, Roma 2001. Su Giovanni Paolo II e i movimenti: L. Moreira Neves, I movimenti ecclesiali nel magistero di Giovanni Paolo II in I movimenti nella Chiesa: atti del 2. Colloquio internazionale su Vocazione e missione dei laici nella Chiesa oggi. Rocca di Papa, 28 febbraio - 4 marzo 1987, Nuovo Mondo, Milano 1987. 
anche alle chiese locali, anche ai vescovi, che non è loro consentito indulgere ad alcuna pretesa d'uniformità assoluta nelle organizzazioni e programmazioni pastorali. Non possono far assurgere i loro progetti pastorali a pietra di paragone di quel che allo Spirito Santo è consentito operare.... Non è lecito pretendere che tutto debba inserirsi in una determinata organizzazione dell'unità; meglio meno organizzazione e più Spirito Santo! Soprattutto non si può sostenere un concetto di comunione in cui il valore pastorale supremo consista nell'evitare conflitti. La fede è sempre anche spada e può esigere proprio il conflitto per amore di verità e di carità (cf. Mt 10, 34). Un progetto di unità ecclesiale in cui i conflitti fossero liquidati a priori come polarizzazione e la pace interna fosse ottenuta a prezzo della rinuncia alla totalità della testimonianza, ben presto si rivelerebbe illusorio. Non è lecito, infine, che s'instauri un certo atteggiamento di superiorità intellettuale per cui si bolli come fondamentalismo lo zelo di persone animate dallo Spirito Santo e la loro candida fede nella Parola di Dio, e non si consenta nient'altro che un modo di credere per il quale il "se" e il "ma" sono più importanti della sostanza di quanto si dice di credere. ${ }^{20}$

Ecco che dunque la storia antica, come quella più recente, ci insegna come in ogni momento di difficoltà, in cui qualcosa di nuovo nasce o cambia, non serve imporre con la forza dell'istituzione la continuità del passato. Occorre cultura (quella di Atanasio e quella del Card. Ratzinger), apertura all'azione dello Spirito e lungimiranza, per creare le condizioni perché tutti gli elementi costitutivi del popolo di Dio, realtà complessa e variegata e non monolitica, possano liberamente e responsabilmente svolgere la propria missione unica che è quella di costruire il Regno di Dio e annunciarne la venuta a tutti. Nessuno può assolutizzare se stesso, il proprio gruppo o la propria parte di verità, ma deve essere al servizio di una comunione che non è unità nell'uniformità, ma pluralità delle espressioni storiche dello Spirito. Esse, se sono veramente tali sia nel laicato come nel clero, non potranno che avere a cuore la comunione di tutti gli uomini nell'unica famiglia dei figli di Dio.

Parole chiavi: clero, laicato, Chiesa dei primi secoli, monachesimo egiziano, Chiesa istituzionale, carisma.

\section{Bibliografia:}

1. Athanase d'Alexandrie, Vie d'Antoine, a cura di G. J. M. Bartelink, (SC 400), Édions du Cerf, Paris 1994.

2. Bartelink G. J. M., Les rapports entre le monachisme égyptien et l'episcopat d'Alexandrie, in Alexandrina. Hellénisme, Judaïsme et christianisme à Alexandrie. Mélanges offerts au P. Claude Mondésert, Étitions du Cerf, Paris 1987.

20 Pontificio Consiglio per i Laici, Atti del Congresso mondiale dei movimenti ecclesiali, Roma, 27-29 maggio 1998, LEV, Città del Vaticano 1999. 
3. Brennan B., Athanasius' Vita Antonii, a Sociological Interpretation, in Vigiliae Christianae 39, Leiden 1985, pp. 209-227.

4. Brennecke H. C., et al. (edd.), Athanasius Werke, vol. II, 8, Walter De Gruyter, Berlin-New York 2000.

5. Ciola N., Indicazioni bibliografiche sulla teologia del laicato, in Orientamenti Pastorali, 34 (1986), pp. 79-82.

6. Colombàs G. M., Il monachesimo delle origini, Jaca Book, Milano 1984-1990.

7. Concilio Ecumenico vaticano II, Decreto sull'apostolato dei Laici Apostolicam Actuositatem.

8. Congar Y.-M., Laïc et laïcat, in Dictionnaire de Spiritualité, IX, Paris 1976, coll. 79-108.

9. Faivre A., Les Fonctions ecclésiales dans les écrits Pseudo-Clémentins, in "Revue de Sciences Religieuses", 50, Strasbourg 1976, pp. 97-111.

10. Frend W. H. C., I monaci e la fine del paganesimo greco-romano in Siria e in Egitto, in P. F. Beatrice (a cura di), Lintolleranza cristiana nei confronti dei pagani, EDB, Bologna 1990, pp. 37-55.

11. Goldie R., Laici, laicato, laicità. Bilancio di trent'anni di bibliografia, Ed. AVE, Roma 1986.

12. H. Hoppenbrouwers, La plus ancienne version latine de la vie de S. Antoine par S. Athanase, Nijmegen 1960.

13. Hegge C., Il Vaticano II e i movimenti ecclesiali: una recezione carismatica, Città Nuova, Roma 2001.

14. M. Alexandre, La costruction d'un modèle de sainteté dans la Vie d'Antoine par Athanase d'Alexandrie, in P. Walter, Saint Antoine entre mythe et légende, Ellug, Grenoble 1996, pp. 63-93.

15. Moreira Neves L., I movimenti ecclesiali nel magistero di Giovanni Paolo II in I movimenti nella Chiesa: atti del 2. Colloquio internazionale su Vocazione e missione dei laici nella Chiesa oggi. Rocca di Papa, 28 febbraio - 4 marzo 1987, Nuovo Mondo, Milano 1987.

16. Neuner P., Per una teologia del popolo di Dio, Queriniana, Brescia 2016.

17. Pontificio Consiglio per i Laici, Atti del Congresso mondiale dei movimenti ecclesiali, Roma, 27-29 maggio 1998, LEV, Città del Vaticano 1999.

18. Rapp C., Holy Bishops in Late Antiquity. The Nature of Christian Leadership in an Age of Transition, Berkeley 2005.

19. Rehm V. B., Irmscher J., Paschke F. (a cura di), Die Pseudoklementinen I. Homilien, $2^{\text {nd }}$ ed., (Die griechischen christlichen Schriftsteller 42), Berlin, Akademie-Verlag 1969, p. 5-22.

20. Scola A., Giuliodori C., et al. (a cura di), Il laicato. Rassegna bibliografica in lingua italiana, tedesca, francese, LEV, Città del Vaticano 1987.

21. Williams M. A., The Life of Antony and the Domestication of Charismatic Wisdom in Thematic Studies, Charisma and Sacred Biography, in "The Journal of the American Academy of Religion" 48, Oxford 1982, pp. 23-40.

22. Wipszycka E., La christianisation de l'Égypte aux IVe-Ve siècles: aspects sociaux et ethniques, in ead., Études sur le christianisme dans l'Égypte de l'antiquité tardive, (Studia Ephemeridis Augustinianum 52), Institututm Augustinianum, Roma 1996, pp. 64-105. 